\title{
Effect of wet and dry conditions of MUF polymers on strength properties of treated peat soil
}

\begin{abstract}
Peat soil is one of the abundant soft soil material vastly found in east costal area of Peninsular Malaysia. Soft soil can be treated using wet and dry mixing method for the purpose of deep stabilization method. It is either mixed thoroughly with conventional or nonconventional binder. Among other properties of concern in soil, stabilization is a strength of treated peat medium which is much likely dependant on condition of wet and dry mixture between binder and treated soil. Essentially, nonconventional binder such as Melamine Urea Formaldehyde (MUF) polymer resin has recently found a fresh look in this study intendedly for future possible application, especially for rapid application stabilization technique. This chapter presents the performance of two types of MUF resin, namely MUF powdered (MUF-P) and MUF liquid (MUF-L) resin which were tested to treat peat soil for determination of unconfined compressive strength (UCS), and elasticity modulus. Both type of resins were mixed with peat specimens as wet and dry binder for stipulated periods of curing times (1ï 7 days). Results showed that MUF-P resin had performed significantly to improve UCS of initially high moisture content of treated peat soil specimens for up to $287 \mathrm{kPa}$ in a week. Improvement of elasticity modulus, E50 in term of secant modulus also noticeable within $7 \ddot{i}$ 14 days of curing periods. However, MUF-L has significantly improved UCS of treated specimens particular for air curing application at lower range of moisture content with significant improvement of up to $500 \mathrm{kPa}$ within 7 days curing period.
\end{abstract}

Keyword: Peat soil; Wet condition; Dry condition; Melamine urea formaldehyde; Unconfined compressive strength 